\title{
GCU
}

Glasgow Caledonian

University

University for the Common Good

\section{Optimal beam radius for LED-based indoor positioning algorithm}

Ajmani, Manisha; Sinanovic, Sinan; Boutaleb, Tuleen

Published in:

2016 International Conference for Students on Applied Engineering (ICSAE) Proceedings

DOI:

10.1109/ICSAE.2016.7810217

Publication date:

2017

Document Version

Author accepted manuscript

Link to publication in ResearchOnline

Citation for published version (Harvard):

Ajmani, M, Sinanovic, S \& Boutaleb, T 2017, Optimal beam radius for LED-based indoor positioning algorithm. in ZYA Al-Shibaany \& AF Hameed (eds), 2016 International Conference for Students on Applied Engineering (ICSAE) Proceedings., 7810217, IEEE, pp. 357-361, International Conference for Students on Applied Engineering (ICSAE), 20/10/16. https://doi.org/10.1109/ICSAE.2016.7810217

\section{General rights}

Copyright and moral rights for the publications made accessible in the public portal are retained by the authors and/or other copyright owners and it is a condition of accessing publications that users recognise and abide by the legal requirements associated with these rights.

Take down policy

If you believe that this document breaches copyright please view our takedown policy at https://edshare.gcu.ac.uk/id/eprint/5179 for details of how to contact us. 


\title{
Optimal beam radius for LED-based indoor positioning algorithm
}

\author{
Manisha Ajmani*, Sinan Sinanović* and Tuleen Boutaleb* \\ *School of Engineering and Built Environment, Glasgow Caledonian University, Glasgow, G4 0BA,UK \\ Email: (manisha.ajmani, sinan.sinanovic, t.boutaleb)@gcu.ac.uk
}

\begin{abstract}
Indoor positioning systems have the potential to replicate the success of outdoor positioning systems, but owing to the expensive and less accurate technology currently available for indoor positioning, they have not been able to take-off. LEDbased Visible Light Communication (VLC) systems can solve this problem, but owing to complex algorithms and unoptimized parameter values the desired accuracy has not yet been achieved. This research addresses the problem of overlapping light radiation regions, which leads to lower accuracy in a VLC system, and proposes an algorithm to accurately determine the position of a device with respect to pre-positioned LEDs when it is receiving signal from multiple transmitting LEDs. To check the accuracy of this Optimal Beam Radius Indoor Positioning (OBRIP) algorithm numerous possible positions of a device uniformly distributed in a room with an indoor positioning system have been simulated to calculate the error in position estimation. Also, from the simulations, optimal values for beam radius, for a given number of LEDs in an array, separation between adjacent LEDs in an array for different room shapes has been calculated.
\end{abstract}

Keywords-Visible light communication (VLC); Indoor positioning system (IPS); Light-emitting diode (LED); Infrared (IR); Electromagnetic (EM)

\section{INTRODUCTION}

Outdoor tracking and positioning systems have evolved extensively in the past decade in terms of accuracy and efficiency [1]. GPS has taken the world of outdoor positioning [2] and tracking by storm leading to huge success of applications like Google Maps and Apple Maps. Research in the field of Indoor tracking and positioning has been going on to produce the similar results. The existing indoor positioning systems (IPS) are based on microwave technology or optical tracking and imaging [3]. Indoor positioning system based on microwave technology include Bluetooth, ultra-wide band (UWB) or wireless local area network [4] integrated into the systems. Many IPS devices have been designed using these technologies, but till now there is no clear winner. Some major challenges for the design of an Indoor positioning solution are: 1. Additional infrastructure is required leading to inflated costs, i.e. expensive implementation and installation (IR, Ultrasound, RFID etc.) [5], [6]

2. RF based solutions are not safe for environments like hospitals, airplanes etc. as it may cause, and suffer, from Electromagnetic (EM) interference [6], [7].

3. Indoor positioning requires very high accuracy, which existing technologies like GPS fail to provide [6], [7].
Optical tracking and imaging methods provide good accuracy up to few millimeters [3], but it involves high cost and complex usage of image processing. Visible Light Communication (VLC) using LEDs has emerged as a new solution for indoor positioning systems [8]. VLC is a unique method of highspeed data transfer which can operate both as a lighting source and a communication tool, addressing the problem of additional infrastructure. It is based on the wireless optical communication principle in which the data to be transmitted is modulated according to the visible light emitted by the LED [9]. LEDs offer several advantages including a higher bandwidth, high brightness efficiency and longer life [10], [11] in comparison to traditional light sources, and the VLC system as a whole offers many attractive features such as high accuracy, high bandwidth capacity, robustness to EM interference, excellent security and low cost deployment [12], [13]. VLC technology is also suitable for various other applications like navigation and broadcasting wireless communication [14].

Though VLC can lead to highly accurate position estimation, the existing positioning algorithms have not been able to tap into the potential of this technology in a practical and low complexity manner. These algorithms have proven to be reasonably successful at reducing the error in position estimation and also are relatively inexpensive [15], but they still suffer from the problem of packet loss due to interference created by overlapping light beam regions. In this research this problem of overlapping light beams is used to the system's advantage and a simple and practical positioning algorithm is proposed which helps reduce the anomaly in position estimation to enhance the system accuracy. These low-cost, reliable and highly accurate alternatives to existing indoor position systems could be utilized in the medical sector for monitoring patients, or in large commercial stores to monitor location of staff.

\section{Optimal BeAm RAdiUs Indoor Positioning (OBR IP)}

The proposed algorithm employs an array of LEDs installed in a room at fixed locations. These LEDs constantly transmit their location coordinates in the form of optical signals within the room. When a device (or receiver) enters the room, it detects one or more signals from light beams of different LEDs. If the signal it receives is only from one LED, the coordinates of the LED are assigned the device's reference point. If the device receives multiple signals, i.e. if it is in the 
overlapping region of two or more LEDs, it finds the centroid of the received coordinates and stores it as the reference point. Thus, the overlap region is used as an advantage to increase the accuracy in position estimation because more overlapping region means more reference points. This algorithm has been simulated in MATLAB to analyze its performance. Various random positions of an object in a room with an indoor positioning system are considered and correspondingly the error in position estimation is averaged. Also, various parameters like radius of the beam and number of LEDs in an array have been varied and the output is analyzed in order to obtain optimized values for these parameters. Radius vs. Average Error plot for different radii, Radius vs. Average Error plot for varying number of LEDs have been plotted, and based on these graphs optimized parameter values are determined.

\section{Simulation SETUP AND Results}

In this paper, simulation based procedure is followed to study the performance of the LED-based indoor positioning system.

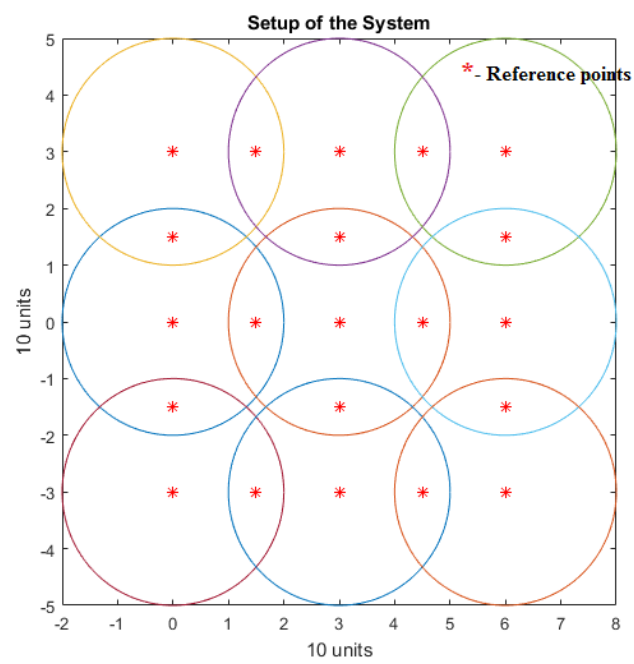

Fig. 1: Simulation of a room with indoor positioning system

In this algorithm, a fixed size of room (10*10 sq. units) is assumed, which contains nine pre-positioned LEDs. The beam area of light radiation of an LED in the array is assumed to be in the form of a circle. This area overlaps for various LEDs in the array and the beam radius of the LEDs is varied to change the area of this overlapping region. Based on the positioned LEDs and overlapping region various reference points are generated and stored (Fig. 1). These reference points are the positions of the LEDs and the midpoint of the overlapping regions. The midpoints of overlapping region are calculated by finding the average of the LEDs positions whose beam area leads to this overlapping region.

To simulate the position of an object in a room 25000 uniformly distributed random points are generated within the room which can be inside the overlapping region, noncoverage region or within beam area of one LED. This large number of random points would cover the entire area of the room. In the first scenario, when the device will receive signal from only one LED, it will be assigned the reference point as position of that LED. In the second scenario, when it will receive signal from multiple LEDs, it will be assigned a reference point as the mean of all LEDs positions it is getting a signal from. In the third scenario, when it receives no signal it will be assigned center of the room as its reference point. The same scenarios have been implemented in this simulation. To check the accuracy of this model the difference between the actual position (coordinates of the random point) and its corresponding reference point was calculated. The average error is calculated for the 25000 simulated random points. The same scenario is repeated for a range of values of the beam radius to find its optimal value. After beam radius optimization, number of LEDs in the array and separation between LEDs in array are varied for different room shapes to find out optimal values.

The following equation is used to calculate the error in position estimation, by finding the distance between the actual and the reference (or allocated) position.

$$
\begin{gathered}
d=\sqrt{\left(x_{1}-x_{2}\right)^{2}+\left(y_{1}-y_{2}\right)^{2}} \\
A v g=S / N
\end{gathered}
$$

where $\mathrm{d}$ is distance between the assigned reference position and actual position,

$\left(x_{1}, y_{1}\right)$ is the reference position and,

$\left(x_{2}, y_{2}\right)$ is the actual position,

$A v g$ is the average error,

$S$ is the sum of the distances between each random point and its reference point,

$N$ is the number of total random point generated.

In our case $N=25000$

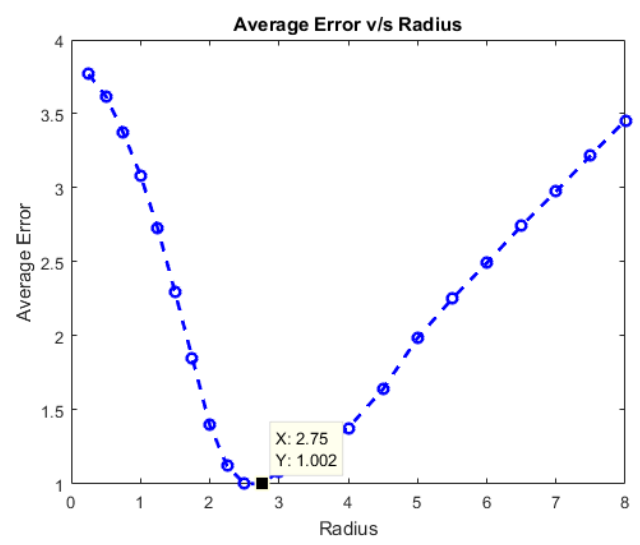

Fig. 2: Beam Radius vs. Average Error in position estimation

\section{A. Variation of beam radius}

Beam radius is the maximum extent up to which a single LED can transmit with the required intensity which the device 
TABLE I. Average error v/s Radius for different No. of LEDS

\begin{tabular}{|c|c|c|}
\hline No. of LEDS & Optimal Radius & Average Error \\
\hline 9 & 2.75 & 1.00 \\
\hline 8 & 2.75 & 1.19 \\
\hline 7 & 2.75 & 1.35 \\
\hline 6 & 2.75 & 1.78 \\
\hline 5 & 2.75 & 2.04 \\
\hline
\end{tabular}

can detect. The radius is varied from 0.5 to 8 units at an interval of 0.25 for parameter optimization. From the simulations, it is observed that the average error varies significantly with the radius (Fig. 2). We were also able to determine optimum beam radius for each LED from the plot between average error and radius of signal transmission (Fig. 2). We note that the error varies asymmetrically around the optimum point with error increasing significantly for smaller radii and only slightly for larger than optimal radius. The optimum radius result can be equivalently represented as a ratio between the light beam coverage area and the area of the room which corresponds to 0.2 in a considered scenario. Average error is minimum i.e. 1.001 when the radius of beam is 2.5

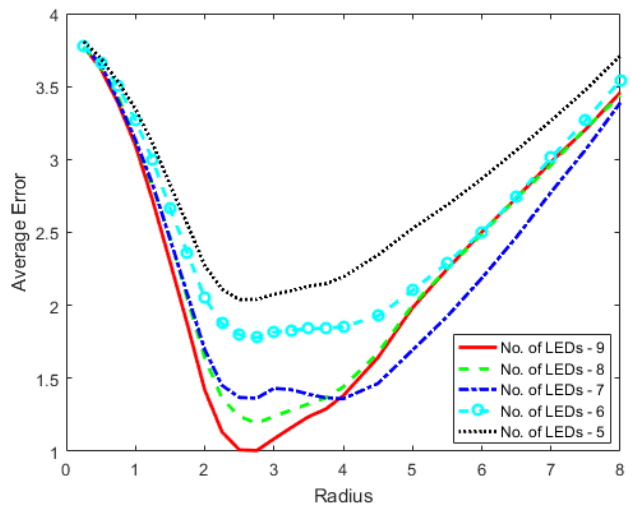

Fig. 3: Beam Radius vs. Average Error in position estimation for different number of LEDs

\section{B. Variation of number of LEDs}

The effect of number of LEDs in an array is observed when number of LEDs are varied from 5 to 9. Initially 9 LEDs are placed in a way that the distance between adjacent LEDs is same, and then LEDs are removed in such a way that their distance from their adjacent LEDs are 3 units horizontally and vertically and $3 \sqrt{2}$ units diagonally. For each LED count, 5 to 9 , the beam radius of each LED is also varied from 0.25 units to 8 units. From the graph (Fig. 3) and TABLE I, it is observed that average error increases as the number of LEDs decreases for each value of beam radius. The best value for number of LEDs is found to be 9, which is expected for this case. As the number of LEDs increases, the number of reference points will increase because the number of overlapping region will be more. This would lead to further decrease in average error in position estimation.

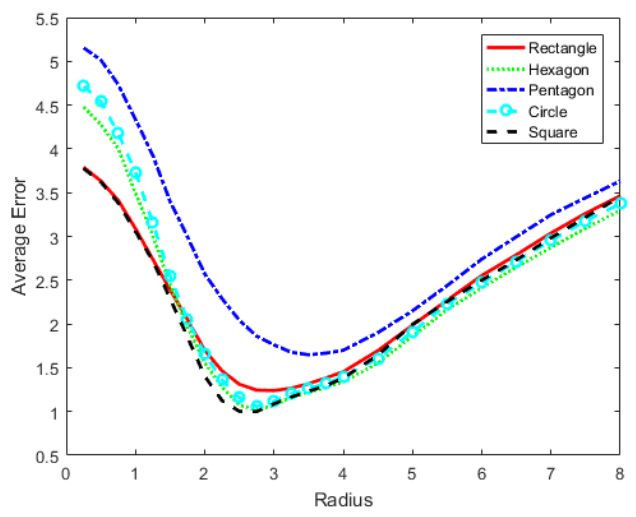

Fig. 4: Beam Radius vs. Average Error in position estimation for different room shape

TABLE II. Average error v/s Radius for different Room Shapes

\begin{tabular}{|c|c|c|}
\hline Room shape & Optimal Radius & Average Error \\
\hline Square & 2.75 & 1.00 \\
\hline Hexagon & 2.75 & 1.02 \\
\hline Circle & 2.75 & 1.05 \\
\hline Rectangle & 2.75 & 1.23 \\
\hline Pentagon & 3.5 & 1.66 \\
\hline
\end{tabular}

\section{Variation of room shape}

For the following room shapes: square (10 units x 10 units), rectangle (11.1 units $\times 9$ units), circle (radius $=5.64$ units), hexagon (radius $=6.21$ units) and pentagon (radius $=7.6$ units) the algorithm was implemented while keeping the room area constant (100 sq. units). The number of LEDs in the array is kept at 9 and the position of the array is such that the position of the middle LED coincides with the geometric center of the shape. It is observed that for each room shape average error in position estimation varies along with the beam radius. From the graph (Fig. 4) and TABLE II, it can be seen that the minimum average error occurs when the shape of the room is square followed by hexagon, circle, rectangle and pentagon. This is the order of effectiveness of the algorithm for different room shapes. It can be seen that regular room shapes like circle, square and hexagon have almost identical low average error, whereas pentagon and rectangle, which are irregular shapes have a higher average error.

\section{Variation of the LEDs grid size}

Separation between adjacent LEDs is also an important parameter to be optimized. In this simulation we vary the separation from 1.5 units to 5.5 units for different room shapes, keeping the position of the center LED fixed i.e. (3,0) (Fig. 5) and observe the change in average error in position estimation. From the graphs (Fig. 6, 8, 10, 12), we can observe that as separation is increased, average error decreases for each room shape.From the observations (Fig. 7, 9, 11, 13), when the separation between adjacent LEDs in the array is increased from 3.5 units to 5.5 units, it can be concluded that the minimum average error obtained is almost same for each 
separation distance, but for different values of beam radius.

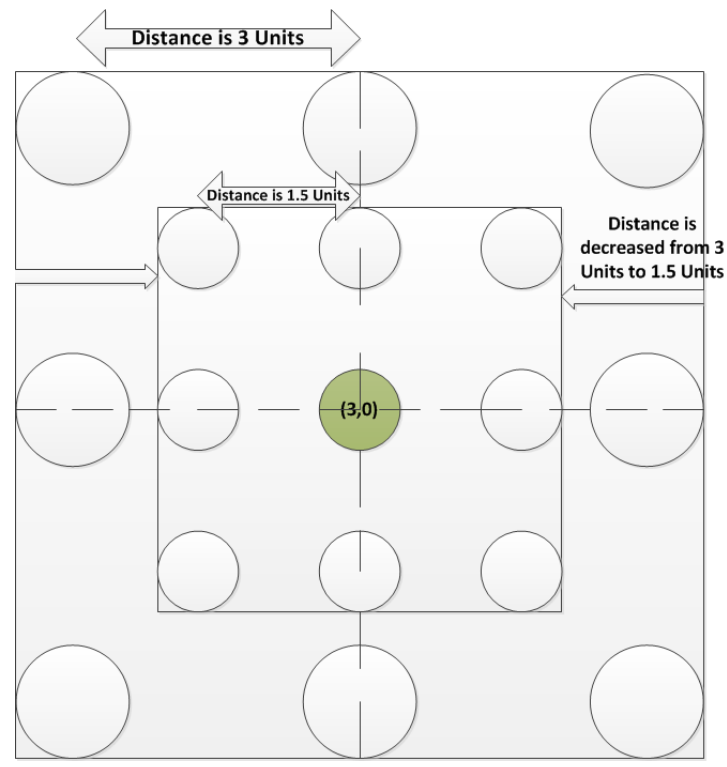

Fig. 5: Simulation of a room for different grid size

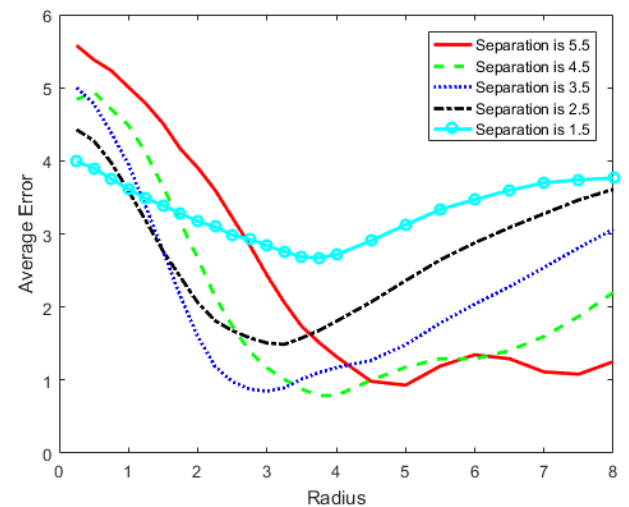

Fig. 6: Average error vs. radius for different grid size (Circle)

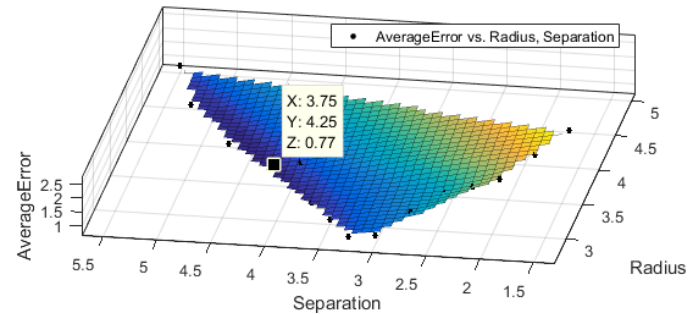

Fig. 7: Average error vs. Radius, Separation (Circle)

\section{CONCLUSION}

The algorithm explained above provides solution to the problem of overlapping light radiation regions and uses it as an advantage to increase accuracy in position estimation. From

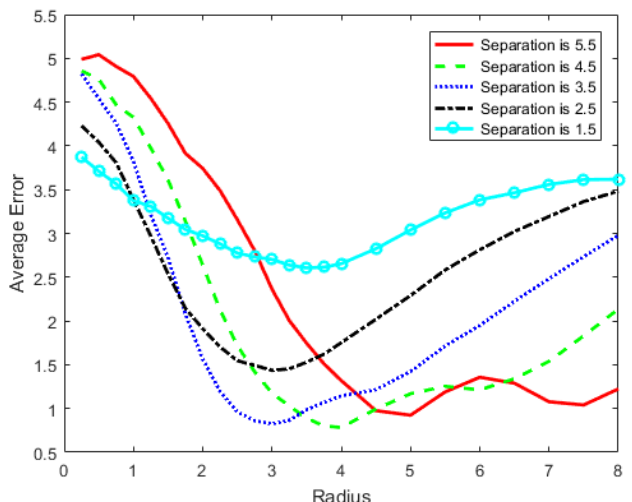

Fig. 8: Average error vs. radius for different grid size (Hexagon)

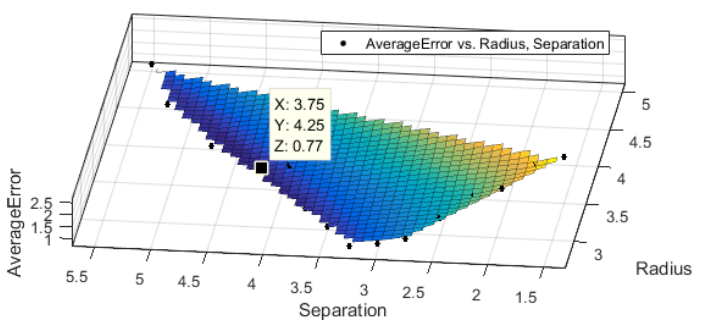

Fig. 9: Average error vs. Radius, Separation (Hexagon)

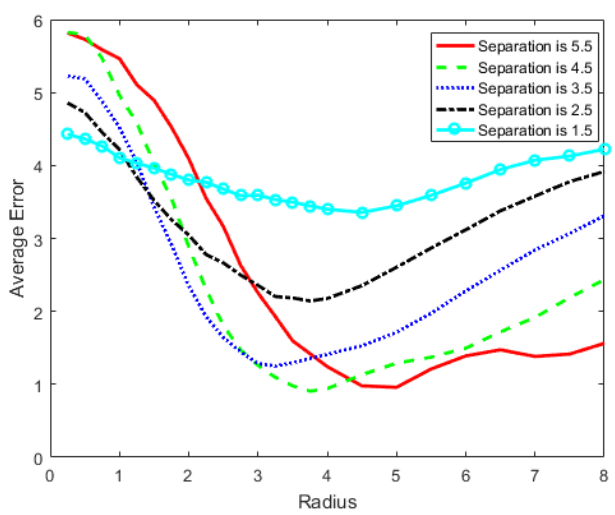

Fig. 10: Average error vs. radius for different grid size (Pentagon)

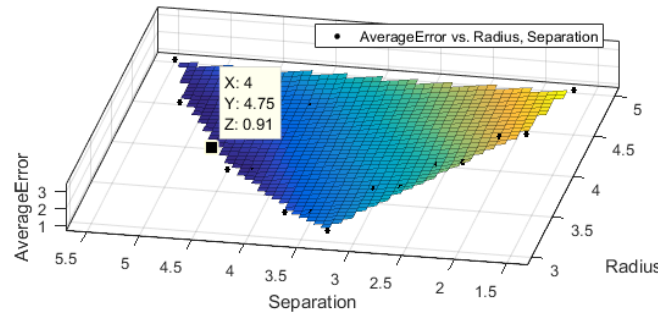

Fig. 11: Average error vs. Radius, Separation (Pentagon) 


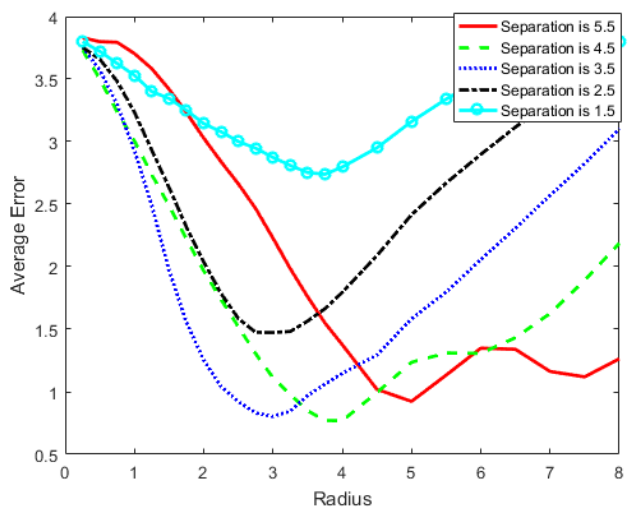

Fig. 12: Average error vs. radius for different grid size (Square)

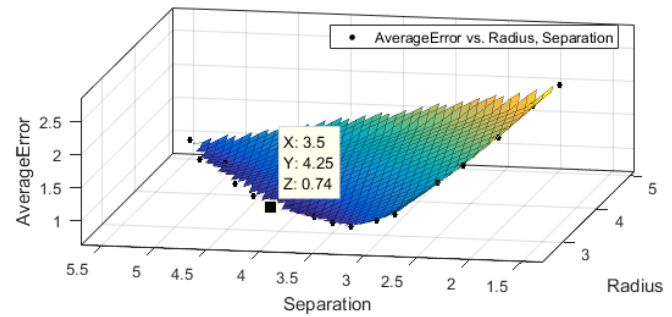

Fig. 13: Average error vs. Radius, Separation (Square)

the simulation experiment we studied scenario with different values for number of LEDs, separation between LEDs and radius of LED beam for various room shapes. These best values are obtained for a fixed room size. Thus, their ratio with the room size can be used as a constant parameter when deciding on values for different room sizes. High accuracy is achieved using this algorithm in the simulations and the same can be tested in practical experiments. To increase the accuracy of this algorithm multiple receivers, instead of one, can be used on one device. This way each receiver would have its own reference point which might further improve the accuracy of this algorithm. Using this algorithm as a foundation, a cheap and accurate alternative to indoor navigation can also be implemented.

\section{ACKNOWLEDGMENT}

The authors would like to thank Glasgow Caledonian University for funding the research, without their help this research would not have been possible. We would also take this opportunity to thank the staff of the School of Engineering and Built Environment at Glasgow Caledonian University for being very cooperative and providing excellent research environment and equipment. We would also like to thank the Graduate School department for conducting 'Research Workshop' which was extremely helpful in writing this research paper. The authors would like to dedicate this research to the people affected by dementia, for whom this research was initiated, and hopefully we would be able to make a difference in their lives with our research.

\section{REFERENCES}

[1] D. Dardari, P. Closas, and P. M. Djurić, "Indoor tracking: Theory, methods, and technologies," IEEE Transactions on Vehicular Technology, vol. 64, no. 4, pp. 1263-1278, 2015.

[2] P. Luo, Z. Ghassemlooy, H. Le Minh, A. Khalighi, X. Zhang, M. Zhang, and C. Yu, "Experimental demonstration of an indoor visible light communication positioning system using dual-tone multi-frequency technique," in Optical Wireless Communications (IWOW), 2014 3rd International Workshop in. IEEE, 2014, pp. 55-59.

[3] S. Yamaguchi, V. V. Mai, T. C. Thang, and A. T. Pham, "Design and performance evaluation of vlc indoor positioning system using optical orthogonal codes," in Communications and Electronics (ICCE), 2014 IEEE Fifth International Conference on. IEEE, 2014, pp. 54-59.

[4] Z. Zhou, M. Kavehrad, and P. Deng, "Indoor positioning algorithm using light-emitting diode visible light communications," Optical Engineering, vol. 51, no. 8, pp. 085009-1, 2012.

[5] A. Yazici, U. Yayan, and H. Yücel, "An ultrasonic based indoor positioning system," in Innovations in Intelligent Systems and Applications (INISTA), 2011 International Symposium on. IEEE, 2011, pp. 585-589.

[6] S. De Lausnay, L. De Strycker, J.-P. Goemaere, N. Stevens, and B. Nauwelaers, "Optical cdma codes for an indoor localization system using vlc," in Optical Wireless Communications (IWOW), 2014 3rd International Workshop in. IEEE, 2014, pp. 50-54.

[7] S.-H. Yang, E.-M. Jung, and S.-K. Han, "Indoor location estimation based on led visible light communication using multiple optical receivers," IEEE Communications Letters, vol. 17, no. 9, pp. 1834-1837, 2013.

[8] S. Babadi, R. Ramirez-Iniguez, T. Boutaleb, and T. Mallick, "Performance analysis of a dtirc-led illumination structure," in Optics and Measurement Conference 2014. International Society for Optics and Photonics, 2015, pp. $944205-944205$.

[9] T. Huang, X. Gao, Y. Guo, S. Li, Q. Li, C. Li, H. Zhu, and Y. Wang, "Visible light indoor positioning fashioned with a single tilted optical receiver," in 2015 14th International Conference on Optical Communications and Networks (ICOCN).

[10] Y. Zheng and M. Zhang, "Visible light communications-recent progresses and future outlooks," in 2010 Symposium on Photonics and Optoelectronics. IEEE, 2010, pp. 1-6.

[11] H.-S. Kim, D.-R. Kim, S.-H. Yang, Y.-H. Son, and S.-K. Han, "An indoor visible light communication positioning system using a rf carrier allocation technique," Journal of Lightwave Technology, vol. 31, no. 1, pp. 134-144, 2013.

[12] M. Aminikashani, W. Gu, and M. Kavehrad, "Indoor positioning with ofdm visible light communications," in 2016 13th IEEE Annual Consumer Communications \& Networking Conference (CCNC). IEEE, 2016, pp. 505-510.

[13] W. Zhang, M. S. Chowdhury, and M. Kavehrad, "Asynchronous indoor positioning system based on visible light communications," Optical Engineering, vol. 53, no. 4, pp. 045 105-045 105, 2014.

[14] S.-H. Yang, E.-M. Jeong, D.-R. Kim, H.-S. Kim, Y.-H. Son, and S.-K. Han, "Indoor three-dimensional location estimation based on led visible light communication," Electronics Letters, vol. 49, no. 1, pp. 54-56, 2013.

[15] O. R. Popoola, F. B. Ogunkoya, W. O. Popoola, R. Ramirez-Iniguez, and S. Sinanovic, "Indoor localization based on multiple leds position estimation," accepted in The 17th International workshop on Signal Processing advances in wireless Communications, SPAWC, 2016. 